\title{
Towards an Efficient and Robust Foot Classification from
}

\section{Pedobarographic Images}

Francisco P. M. Oliveira*, Andreia Sousa ${ }^{\#}$, Rubim Santos”, João Manuel

R. S. Tavares ${ }^{*}$

${ }^{*}$ Faculdade de Engenharia da Universidade do Porto (FEUP)/Instituto de

Engenharia Mecânica e Gestão Industrial (INEGI), Rua Dr. Roberto Frias, 4200-465

Porto, Portugal

${ }^{\#}$ Escola Superior da Tecnologia de Saúde do Porto (ESTSP), Instituto Politécnico do Porto (IPP), Centro de Estudos de Movimento e Actividade Humana (CEMAH), Rua Valente Perfeito 322, 4400-330 Vila Nova de Gaia, Portugal

\section{Corresponding author:}

Professor João Manuel R. S. Tavares

Departamento de Engenharia Mecânica

Faculdade de Engenharia da Universidade do Porto

Rua Dr. Roberto Frias

4200-465 Porto

Portugal

Phone: +351 225081 487, Fax: +351 225081445

Email: tavares@fe.up.pt, url: www.fe.up.pt/ tavares 


\title{
Towards an Efficient and Robust Foot Classification from Pedobarographic Images
}

\begin{abstract}
This paper presents a new computational framework for automatic foot classification from digital plantar pressure images. It classifies the foot as left or right and simultaneously calculates two well-known footprint indices: the Cavanagh's arch index and the modified arch index. The accuracy of the framework was evaluated using a set of plantar pressure images from two common pedobarographic devices. The results were outstanding, since all feet under analysis were correctly classified as left or right and no significant differences were observed between the footprint indices calculated using the computational solution and the traditional manual method. The robustness of the proposed framework to arbitrary foot orientations and to the acquisition device was also tested and confirmed.
\end{abstract}

Keywords: biomechanics; computational methods; image analysis; image alignment; plantar pressure images; footprint indices. 


\section{Introduction}

The functional mechanics of the human foot are greatly influenced by the structure of the foot, in particular, by the medial longitudinal arch height (McCrory et al., 1997). X-rays and ultrasonic devices can easily carry out this measurement; however, they are relatively expensive. Additionally, X-rays imply a potential health risk due to radiation exposure to subjects undergoing scientific or clinical studies.

Footprint parameters have been widely used as a predictor of arch height and foot classification: low arched, normal, and high arched. The study of footprints has numerous applications such as the characterization of populations, the prevention of injuries or the designing of footwear (Cavanagh and Rodgers, 1987; Dowling and Steele, 2001; Forriol and Pascual, 1990; Hernandez et al., 2007; Jung et al., 2001; Staheli et al., 1987). Some of the most widely adopted footprint-based measurements include the arch index (AI) (Cavanagh and Rodgers, 1987), the modified arch index (MAI) (Chu et al., 1995), Staheli's index (Staheli et al., 1987) and Chippaux-Smirak's index (Forriol and Pascual, 1990). In the literature, studies reporting significant correlations between the AI or MAI and the plantar arch height can be found. For instance, considering digital plantar pressure images, Chu et al. (1995) found a significant correlation coefficient ( $r$ ) equal to -0.70 for the AI and the arch height, and of -0.71 for the MAI and the arch height. Also using digital plantar images, other authors (Shiang et al., 1998) found slightly stronger correlation coefficients for AI and MAI and the arch height: -0.73 and -0.74 , respectively. Using walking ink footprints, McCrory et al. (1997) found a similar correlation coefficient $(-0.67)$ between the arch height and AI, and a 
correlation coefficient between the normalized arch height and AI equal to -0.71 was established.

Most of the previous studies concerning the evaluation of footprint indices were based on conventional ink footprints or on a variation. However, the digital plantar pressure images used by Chu et al. (1995) were manually pre-processed, which is prune to errors and of low reproducibility. On the other hand, Shiang et al. (1998) used a fixation device to define the place where subjects should stand to facilitate the processing and analysis of the input images. This solution overcomes foot orientation and localization problems acceptably. However, there are other issues still to be addressed, like the shape and size of each particular foot.

Manual or even semi-automatic procedures to evaluate and compare plantar pressure data in images are somewhat fastidious, very time consuming and can lead to errors associated to the user's skill. Therefore, in recent years, techniques of digital image processing and analysis have been proposed for automated plantar pressure image analysis. Examples include techniques for image matching and analysis (Bastos and Tavares, 2004; Tavares et al., 2000; Tavares and Bastos, 2010), image simulation (Pinho and Tavares, 2004), image registration, i.e. image alignment, (Harrison and Hillard, 2000; Oliveira et al., 2010; Oliveira and Tavares, 2011; Oliveira et al., 2009; Pataky et al., 2008b), and statistical analysis (Pataky et al., 2008a; Pataky and Goulermas, 2008).

The main goal of the present work is to take advantage of those recent techniques of image processing and analysis to build a fully automated computational framework for foot classification and footprint index calculations. As such, the framework should be robust to arbitrary foot orientation, foot type and dimension, and completely independent of the plantar data acquisition device. In this way, the limitations of the 
accompanying software of the common pedobarographic devices could be overcome. Hence, it should be noted that the goal of this paper is not a discussion on footprint indices neither a comparison among them. Nevertheless, for further discussion on this topic see Chu et al. (1995) or Razeghi and Batt (2002). Particularly, an extended review on foot type classification is presented in the latter work; mainly, methods that use visual non-quantitative inspection, anthropometric values, footprint parameters and radiographic evaluation.

\section{Methods}

\subsection{Left/right classification}

The first goal of the developed computational framework is to classify each input image as a left or a right foot. The step by step solution developed can be described as follows:

(1) The algorithm starts by searching for the foot region in the input image, based on the pixel intensities.

(2) Then, the foot is pre-scaled and centered in a square matrix to give it dimensions similar to those of the template images. This size normalization enables feet with different dimensions and defined using distinct pressure sensor arrays to be studied.

(3) In this step, the pre-scaled and centered image is aligned with the template image for the left foot and also with the template image for the right foot. These two alignments are based on the maximization of cross-correlation (Oliveira et al., 2010). (More about the templates images is described in section 2.3.) 
(4) Afterwards, the plantar pressure values of each of the two aligned images are normalized in order to have the same mean pressure as the corresponding template image. This normalization step eliminates the influence of the subject's weight on the image dissimilarity measure computed in the next step.

(5) The sum of the absolute differences (SAD) between each of the two aligned and normalized images and the corresponding template images are computed.

Finally, the input image is classified as a left or a right foot based on the minimal SAD value found.

The flowchart of this classification algorithm is shown in Fig. 1.

[Insert Figure 1 about here]

\subsection{Footprint indices}

\subsubsection{Definitions}

The AI is defined as the ratio between the areas of contact of the different parts of the foot, excluding the toes. Thus, it is given by the ratio of the area of the middle onethird of the footprint to the entire area, Fig. 2:

$$
\mathrm{AI}=\frac{\text { Area of } B}{\text { Area of }(A+B+C)}
$$

To divide the toeless foot into the three regions $(A, B$ and $C)$ in Eq. 1, the line from the tip of the second toe to the center of the heel, commonly known as "foot axis", needs to be defined (Fig. 2). Afterwards, the toeless length $(L)$ can be measured, and the borders of the regions can be drawn perpendicularly to the foot axis, so as the width of each region is $L / 3$ (Cavanagh and Rodgers, 1987). 
[Insert Figure 2 about here]

The MAI is quite similar to AI. The foot is divided into the same regions (Fig. 2), but, instead of computing the ratio among the areas, the ratio of the sum of the pressures presented in the three regions is computed (Chu et al., 1995):

$$
\mathrm{MAI}=\frac{\sum_{i \in B} f_{i}}{\sum_{i \in A \cup B \cup C} f_{i}},
$$

where $f_{i}$ is the pressure denoted by pixel $i$ of image foot $f$.

\subsubsection{Computation of footprint indices}

To compute the footprint indices from an input image, the developed framework starts from the corresponding aligned and classified image and considers the original pixel intensities. Thus, the foot under analysis has a localization, orientation and size similar to the associated image template, but preserving the plantar pressure values. The footprint algorithm calculation can be divided into the following steps:

(1) Image binarization: The pixels with an intensity higher than a threshold value are set to 1 (one) and the remainder to 0 (zero). This threshold value is defined in function of the minimum pressure value that the plantar pressure device used can measure. For example, in the experimental evaluation described in section 2.5 the threshold value was set to $10 \mathrm{kPa}$ for the images acquired by the EMED system and equal to $0.7 \mathrm{~N} / \mathrm{cm}^{2}$ for the ones acquired using the Footscan system.

(2) Toe removal: First, using a rough mask previously built from the associated template image (see section 2.3), most of the input image pixels of the toes are removed, with only the ones nearest the toeless forefoot remaining (region $A$, 
Fig. 2). Then, in a fine mode, based on a local search in the region in which the toes possibly join the forefoot, the remaining pixels of the toes are removed by comparing the intensity of each pixel with the intensities of its neighbors.

(3) Toeless foot segmentation: After the toe removing process, the length of the toeless foot is determined and the foot is divided into the characteristic regions $A$, $B$ and $C$ (Fig. 2). It is important to notice that, as the template image was defined in such a way that its foot axis is parallel to the $y$-axis, then consequently the foot axis of the aligned image is also parallel to the $y$-axis. Thus, the lines that limit the three characteristic regions of the foot are parallel to the $x$-axis and, therefore facilitate any further assessment processes.

(4) Calculation of indices: Since the image under evaluation is suitability binarized and segmented into the toes and the three characteristic regions ( $A, B$ and $C$ ), the computation of the AI and MAI is straightforward using Eq. (1) and (2).

The flowchart of this foot segmentation and footprint index computation algorithm is presented in Fig. 3.

[Insert Figure 3 about here]

\subsection{Image templates}

As previously indicated, two template images are used to align and normalize the plantar pressure image under analysis: one for the right foot and another one for the left foot. It should be noted that these template images only need to be defined once for the entire population under study; however, they should be appropriately representative of the expected pressure distribution. 
In this work, the template image for the right foot was selected from the experimental data set in order to address a normal plantar pressure distribution. Then, the selected image was rotated so that the axis orientation of the represented foot would be parallel to the $y$-axis image (Figure 4). Afterwards, the left foot template image was defined by mirroring the template image of the right foot.

Simultaneously, to assist the toe removal process, a binary image mask was built from the template image for the right foot. Hence, this image mask was allotted the value 0 (zero) in the regions that do not belong to the aligned toeless foot, and 1 (one) for the reminding regions, including the border region between the toes and the toeless foot, Figure 4. The image mask for the left foot was obtained by mirroring the image mask previously built.

These four reference images were then integrated in the computational framework and were successfully used in all the experimental tests that were carried out. They are described and discussed in the following sections.

[Insert Figure 4 about here]

\subsection{Implementation}

The proposed computational framework was fully implemented in $\mathrm{C}^{++}$, using Microsoft Visual Studio 8, and tested on a notebook PC with an AMD Turion 642.0 GHz microprocessor, 1.0 GB of RAM and running Microsoft Windows XP.

\subsection{Data set}

The data set was made up of 122 plantar pressure images: 10 peak pressure images acquired using a 0.5 m Footscan system (RSscan, Olen, Belgium) and 112 plantar 
pressure images acquired using an EMED system (Novel GmbH, Germany). The data set contained plantar pressure images from low arched, normal, and high arched feet. The data from the Footscan system was from 10 subjects ( 4 females, 6 males; 30.1 \pm 7.4 years). The original images were vertically stretched by a factor of 1.5 to correct for non-square sensor array spacing $(5.08 \times 7.62 \mathrm{~mm} /$ sensor $)$.

The data acquired by the EMED system included 56 peak pressure images and 56 static pressure images from 7 men (18.4 \pm 0.5 years) and 21 women (20.4 \pm 2.3 years). The static images were randomly chosen from the plantar pressure image sequences acquired from the subjects when in a static position. The EMED system used has a spatial resolution of 2 sensors per $\mathrm{cm}^{2}$.

\subsection{Accuracy assessment}

To assess the accuracy of the left/right classification, the results obtained by the computational framework were compared to the traditional manual method results.

Two kinds of experiments were carried out: 1) Visual evaluation of the obtained results; namely, analyzing the results of feet alignment and segmentation for each of the 122 plantar pressure images. 2) Comparison between the AI values obtained using the computational framework and the ones obtained by using the traditional manual method. In this comparison, 17 static plantar pressure images from right feet and 17 static plantar pressure images from left feet of 17 subjects were randomly chosen from the data set used. The manual evaluation of the AI was carried out by two individuals trained for the task, after printing each foot image on a sheet in real size. To assess the robustness of the computational framework to arbitrary foot orientations, all the 122 plantar pressure images were successively rotated $90^{\circ}, 180^{\circ}$ 
and $270^{\circ}$, and then each rotated image was classified, in terms of representing a left or right foot, and the associated AI was calculated.

Since the differences between the AI values obtained by the manual method and the ones obtained by the computational framework follow a normal distribution, a One Sample $t$-test was carried out to statistically evaluate the differences between the AI values. The null hypothesis was tested to verify if the mean difference $(\bar{\Delta})$ between them is zero:

$$
\begin{aligned}
& H_{0}: \mu_{\Delta}=\Delta_{0}, \\
& H_{1}: \mu_{\Delta} \neq \Delta_{0} \text {, } \\
& t=\frac{\bar{\Delta}-\Delta_{0}}{S_{\Delta} / \sqrt{N}}, \\
& \text { where } \Delta_{0}=0 \text { and } N=34 \text {. }
\end{aligned}
$$

\section{Results}

\subsection{Accuracy of the left/right classification}

All 122 plantar pressure images were correctly classified, either using the original images or using the rotated images.

\subsection{Accuracy of the arch index computation}

Visual inspection confirmed that all plantar pressure images (both original and rotated) were properly segmented into the four regions: toes, forefoot, arch and heel. Figure 5 shows four examples representing different foot shapes and the corresponding segmentations. 
The differences between the AI values manually and automatically calculated from the 34 static plantar pressure images under evaluation are given in Table 1. It should be noted that, in the case of the traditional manual method the AI values were obtained by averaging the corresponding values calculated by the two evaluators. For a significance of $\alpha=0.05$, the critical value for the statistical $t$-test used is $t_{33}(0.05)=1.69$. Since for all tests we had $t<1.69$ (Table 2), we concluded that $H_{0}$ is accepted, i.e. there is no significant statistical difference between the AI values calculated manually and those given by the computational framework. Consequently, these results also prove the robustness of the framework to arbitrary foot orientations.

[Insert Tables 1 and 2 about here]

\subsection{Processing time}

The average processing time required by the framework to classify and compute AI and MAI values of each of the 34 tested images was around 125 ms. All operational times from the initial reading of the images, the image processing and calculations to the saving of the results were taken into consideration.

\section{Discussion}

Concerning the left/right foot image classification, the results show that the computational framework is efficient, accurate and robust to arbitrary foot shapes and orientations. Additionally, the framework calculated the AI from the digital foot 
images very efficiently and the small differences compared with the manual evaluations (Tables 1 and 2) were not statistically significant. The differences between the AI values of the original and rotated images obtained by the proposed computational framework were also insignificant (Tables 1 and 2), which means that the framework is very robust to arbitrary foot orientations.

A comparison between the MAI values manually calculated versus the ones computed by the computational framework was not carried out. However, since this index is computed from the same regions used for AI and the values of the pixels used are maintained by the image transformations applied, the conclusions for AI are also valid for MAI.

At a first glance, based on the AI values in Table 1 it appears that the population in this study has predominantly low arch feet. However, the main reason for so small AI values is that these values were obtained from plantar pressure images of subjects in a static position and, consequently, the middle foot / plantar pressure device contact is weaker than when the subjects are walking over it.

Alignment quality is important for the accuracy of footprint indices. Therefore, at the initial development stage, different alignment strategies were tried out. The input foot image was aligned with one image template for each foot type: low arched, normal and high arched. Then, the alignment that led to the lowest SAD value was chosen. However, the experimental tests showed that similar results could be achieved using just the template image of the normal foot. Thus, since the developed framework should be as fast as possible, the simpler approach was adopted. Also in order to increase the framework accuracy, we tried out a more accurate alignment algorithm (Oliveira and Tavares, 2011) than the cross-correlation based algorithm used in the tests here. Although the alignment quality was slightly improved, the AI values 
remained almost unaffected. Hence, also based on the requisite for high computational speed, we chose the cross-correlation based alignment algorithm (Oliveira et al., 2010).

We chose the AI and MAI footprint indices because they are frequently adopted in foot classification. However, other footprint/pressure indices or statistical measurements can easily be assessed in an automated way from the aligned and segmented feet images.

Finally, it should be pointed out that the robustness of the proposed computational framework to arbitrary foot orientations, shapes and dimensions, and its independence to the plantar pressure acquisition device carry significant advantages over the traditional methods and solutions. Hence, with the proposed computational framework, the study/characterization of the plantar pressure distribution of large populations can be easily, efficiently and robustly achieved, since no particular requirements are imposed in terms of foot orientation or characteristics of the devices.

\section{Acknowledgements}

This work was partially done under the scope of the following research projects "Methodologies to Analyze Organs from Complex Medical Images - Applications to the Female Pelvic Cavity”, “Cardiovascular Imaging Modeling and Simulation SIMCARD” and “Aberrant Crypt Foci and Human Colorectal Polyps: Mathematical Modelling and Endoscopic Image Processing”, with the references PTDC/EEACRO/103320/2008, UTAustin/CA/0047/2008 and UTAustin/MAT/0009/2008, respectively, financially supported by FCT - Fundação para a Ciência e a Tecnologia in Portugal. 
The first author would like to thank Fundação Calouste Gulbenkian, in Portugal, for his $\mathrm{PhD}$ grant.

\section{References}

Bastos LF, Tavares JMRS, 2004. Improvement of modal matching image objects in dynamic pedobarography using optimization techniques. In: Perales, FJ and Draper, BA (Eds.), Articulated Motion And Deformable Objects - Lecture Notes in Computer Science, Vol. 3179/2004. Springer Verlag, Germany, p. 39-50.

Cavanagh PR, Rodgers MM. 1987. The arch index: a useful measure from footprints. Journal of Biomechanics 20(5):547-551.

Chu WC, Lee SH, Chu W, Wang T-J, Lee M-C. 1995. The use of arch index to characterize arch height: a digital image processing approach. IEEE Transactions on Biomedical Engineering 42(11):1088-1093.

Dowling AM, Steele JR, 2001. What are the effects of gender and obesity on foot structure in children? In Proceedings of the 5th Symposium on Footwear Biomechanics, Zurich, Switzerland, p. 30-31.

Forriol F, Pascual J. 1990. Footprint analysis between three and seventeen years of age. Foot Ankle 11(2):101-104.

Harrison AJ, Hillard PJ. 2000. A moment-based technique for the automatic spatial alignment of plantar pressure data. Proceedings of the Institution of Mechanical Engineers, Part H: Journal of Engineering in Medicine 214(3):257-264.

Hernandez AJ, Kimura LK, Laraya MHF, Fávaro E. 2007. Calculation of Staheli's plantar arch index and prevalence of flat feet: a study with 100 children aged 5-9 years. Acta Ortopédica Brasileira 15(2):68-71. 
Jung S, Lee S, Boo J, Park J, 2001. A classification of foot types for designing footwear of the Korean elderly. In Proceedings of the 5th Symposium on Footwear Biomechanics, Zurich, Switzerland, p. 48-49.

McCrory JL, Young MJ, Boulton AJM, Cavanagh PR. 1997. Arch index as a predictor of arch height. The Foot 7:79-81.

Oliveira FPM, Pataky TC, Tavares JMRS. 2010. Registration of pedobarographic image data in the frequency domain. Computer Methods in Biomechanics and Biomedical Engineering 13(6):731-740.

Oliveira FPM, Tavares JMRS. 2011. Novel framework for registration of pedobarographic image data. Medical \& Biological Engineering \& Computing 49(3): 313-323.

Oliveira FPM, Tavares JMRS, Pataky TC. 2009. Rapid pedobarographic image registration based on contour curvature and optimization. Journal of Biomechanics 42(15):2620-2623.

Pataky TC, Caravaggi P, Savage R, Parker D, Goulermas JY, Sellers W, Crompton RH. 2008a. New insights into the plantar pressure correlates of walking speed using pedobarographic statistical parametric mapping. Journal of Biomechanics 41(9):19871994.

Pataky TC, Goulermas JY. 2008. Pedobarographic statistical parametric mapping (pSPM): a pixel-level approach to foot pressure image analysis. Journal of Biomechanics 41(10):2136-2143.

Pataky TC, Goulermas JY, Crompton RH. 2008b. A comparison of seven methods of within-subjects rigid-body pedobarographic image registration. Journal of Biomechanics 41(14):3085-3089. 
Pinho RR, Tavares JMRS, 2004. Dynamic pedobarography transitional objects by Lagrange's equation with FEM, modal matching and optimization techniques. In: Campilho, AC and Kamel, MS (Eds.), Image Analysis and Recognition - Lecture Notes in Computer Science, Vol. 3212/2004. Springer, Germany, p. 92-99. Razeghi M, Batt ME. 2002. Foot type classification: a critical review of current methods. Gait and Posture 15:282-291.

Shiang T-Y, Lee S-H, Lee S-J, Chu WC. 1998. Evaluating different footprint parameters as a predictor of arch height. IEEE Engineering in Medicine and Biology:62-66.

Staheli LT, Chew DE, Corbett M. 1987. The longitudinal arch. A survey of eight hundred and eighty-two feet in normal children and adults. The Journal of Bone and Joint Surgery 69-A(3):426-428.

Tavares JMRS, Barbosa JMG, Padilha AJ, 2000. Matching image objects in dynamic pedobarography. In Proceedings of the 11th Portuguese Conference on Pattern Recognition (RecPAD 2000), Porto, Portugal.

Tavares JMRS, Bastos LF, 2010. Improvement of modal matching image objects in dynamic pedobarography using optimization techniques. In: Bunke, H, Villanueva, JJ, Sánchez, G and Otazu, X (Eds.), Progress in Computer Vision and Image Analysis. World Scientific, Singapore, p. 339-368. 


\section{TABLE CAPTIONS}

Table 1. Mean AI values and differences between the AI values obtained by the manual method and the proposed computational framework.

Table 2. Statistical test values of the differences between AI values obtained by the manual method and the proposed computational framework, considering the null hypothesis $H_{0}: \mu_{\Delta}=0$ and the One Sample t-Test $t=\frac{\bar{\Delta}-0}{S_{\Delta} / \sqrt{34}}$. 


\section{FIGURE CAPTIONS}

Figure 1. Diagram of the left/right foot classification algorithm.

Figure 2. Original plantar pressure image (on the left), the corresponding aligned and normalized plantar pressure image (in the center) and the segmented three characteristic foot regions plus toes from the normalized image (on the right).

Figure 3. Diagram of the foot segmentation and arch indices computation algorithm.

Figure 4. Image template for right foot (on the left) and image mask used for rough toe removal (on the right).

Figure 5. Four examples of foot normalization and segmentation: the original foot (on the left); the normalized foot in terms of orientation, localization and size (in the middle left); the toeless region with its contour (in the middle right); the segmented foot (on the right). The plantar pressure images of the first row were acquired using the Footscan system, and the images of the other rows were acquired using the EMED system. 
TABLES

Table 1

\begin{tabular}{|c|c|c|}
\hline \multicolumn{3}{|l|}{ Arch index } \\
\hline & Mean & STD \\
\hline $\mathrm{AI}\{$ manual $\}$ & 0.059 & 0.081 \\
\hline AI framework (original images) $\}$ & 0.060 & 0.080 \\
\hline AI $\left\{\right.$ framework (rotated images: $\left.\left.90^{\circ}\right)\right\}$ & 0.060 & 0.080 \\
\hline AI $\left\{\right.$ framework (rotated images: $\left.\left.180^{\circ}\right)\right\}$ & 0.059 & 0.080 \\
\hline AI $\left\{\right.$ framework (rotated images: $\left.\left.270^{\circ}\right)\right\}$ & 0.059 & 0.080 \\
\hline \multicolumn{3}{|l|}{ Arch index differences } \\
\hline & Mean $(\bar{\Delta})$ & $\begin{array}{l}\text { STD } \\
\left(S_{\Delta}\right)\end{array}$ \\
\hline $\mathrm{AI}\{$ manual $\}-\mathrm{AI}\{$ framework (original images) $\}$ & 0.0007 & 0.0073 \\
\hline AI $\{$ manual $\}-\mathrm{AI}\left\{\right.$ framework (rotated images: $\left.\left.90^{\circ}\right)\right\}$ & 0.0007 & 0.0076 \\
\hline $\mathrm{AI}\{$ manual $\}-\mathrm{AI}\left\{\right.$ framework (rotated images: $\left.\left.180^{\circ}\right)\right\}$ & 0.0006 & 0.0072 \\
\hline $\mathrm{AI}\{$ manual $\}-\mathrm{AI}\left\{\right.$ framework (rotated images: $\left.\left.270^{\circ}\right)\right\}$ & 0.0007 & 0.0075 \\
\hline
\end{tabular}


Table 2

\begin{tabular}{|l|l|}
\hline & $t$ \\
\hline AI $\{$ manual $\}$ vs AI $\{$ framework (original images) $\}$ & 0.016 \\
\hline AI $\{$ manual $\}$ vs AI $\left\{\right.$ framework (rotated images: $\left.\left.90^{\circ}\right)\right\}$ & 0.016 \\
\hline AI $\{$ manual $\}$ vs AI $\left\{\right.$ framework (rotated images: $\left.\left.180^{\circ}\right)\right\}$ & 0.014 \\
\hline AI $\{$ manual $\}$ vs AI $\left\{\right.$ framework (rotated images: $\left.\left.270^{\circ}\right)\right\}$ & 0.015 \\
\hline
\end{tabular}




\section{FIGURES}

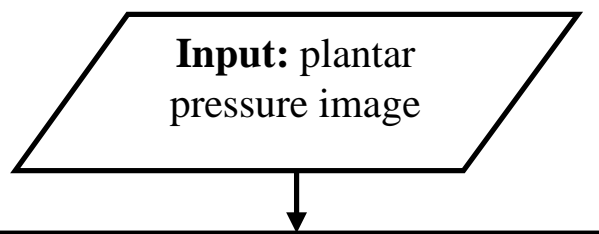

Search for the minimal rectangle that contains the foot and rescale it to the same dimensions of the corresponding rectangle of the template images

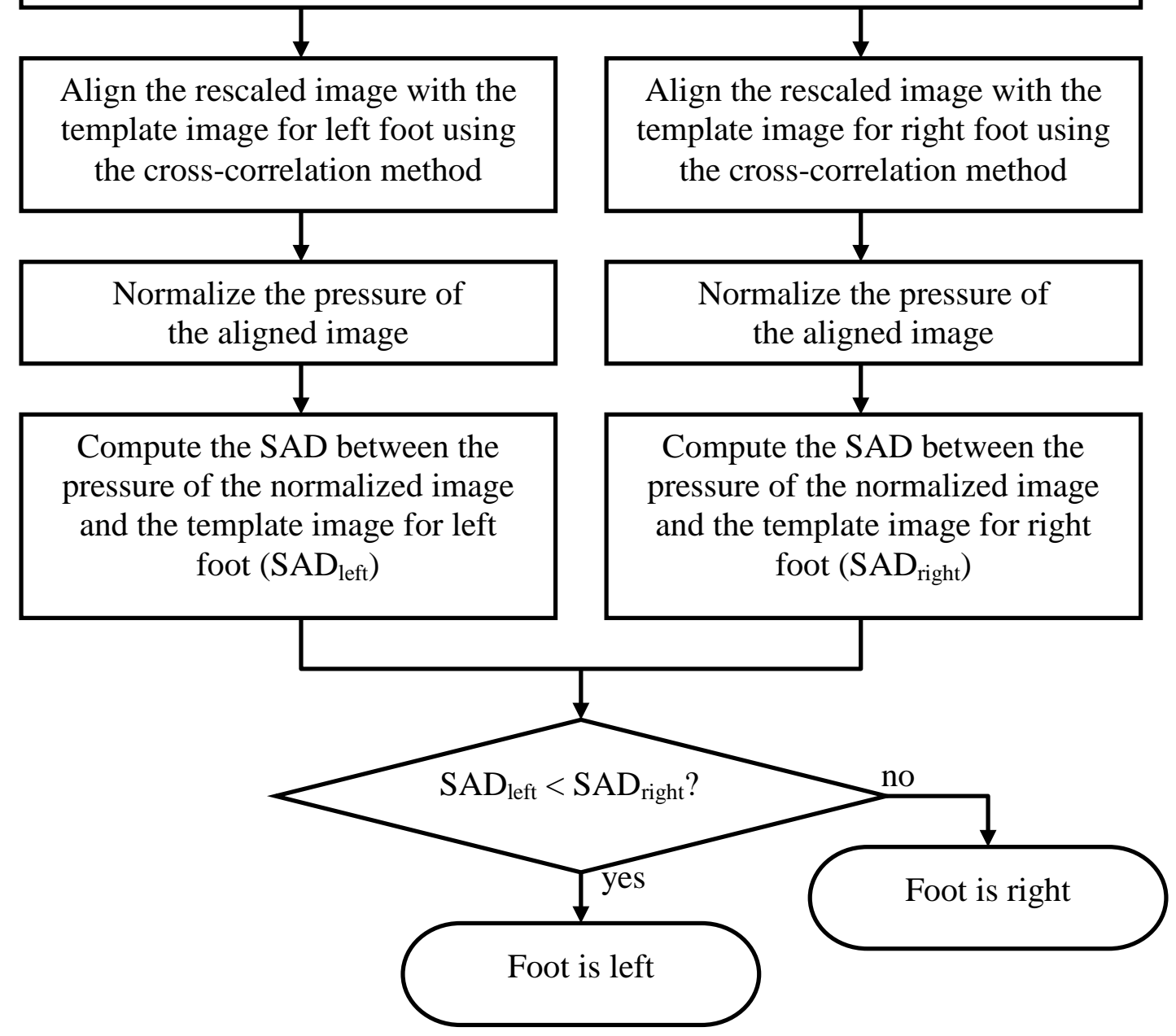

Figure 1 


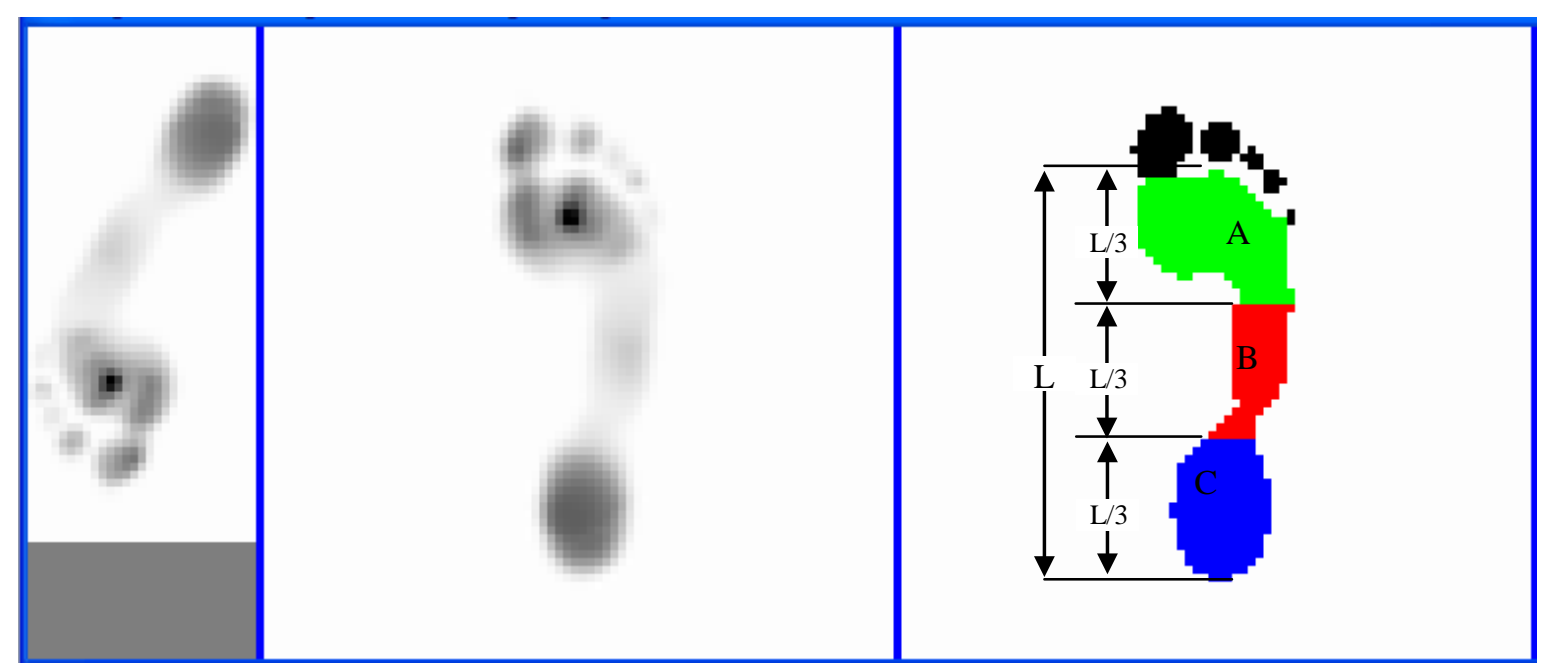

Figure 2 


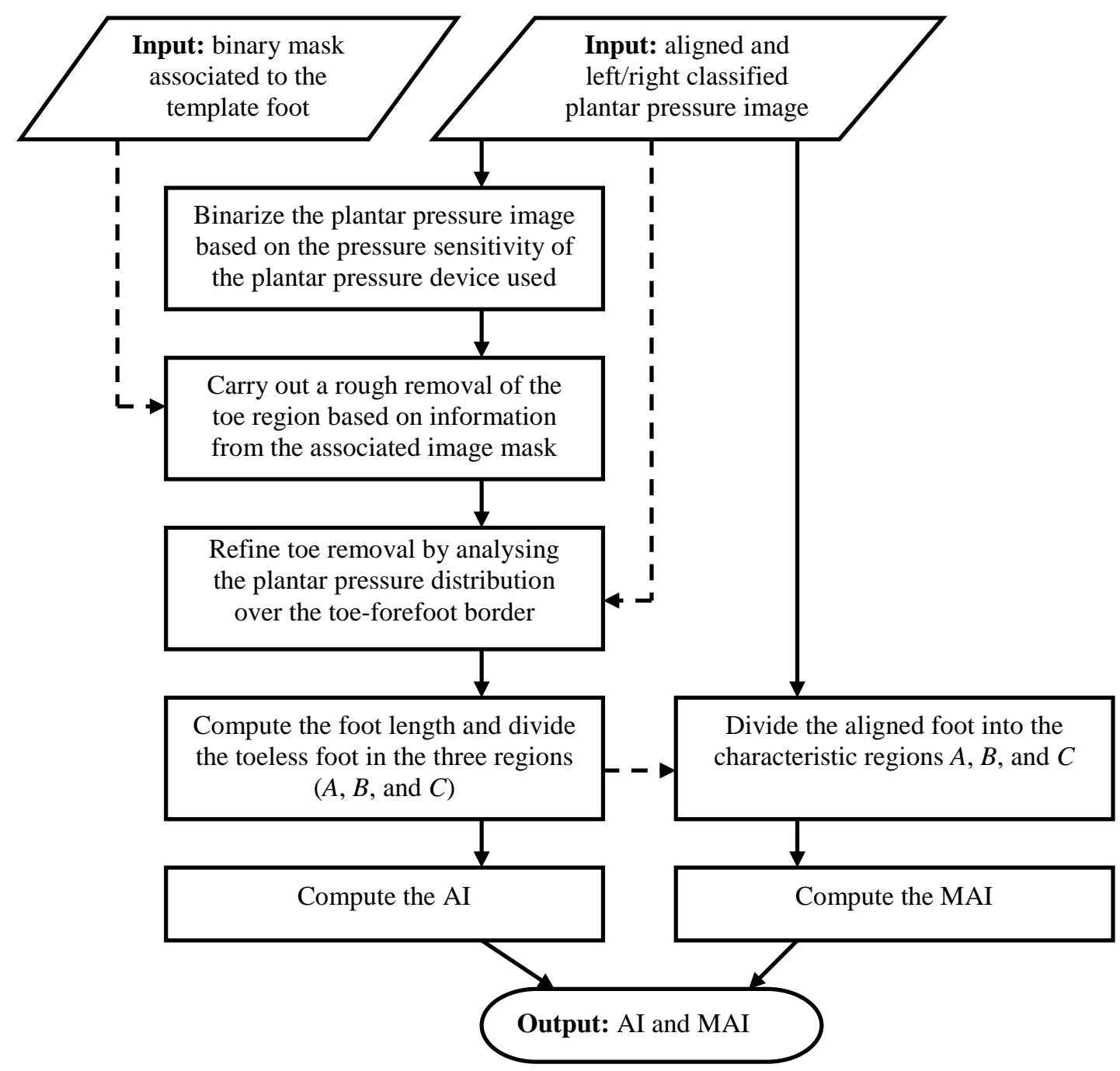

Figure 3 


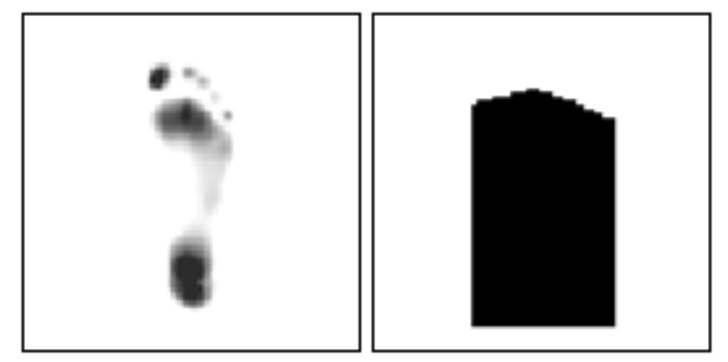

Figure 4 

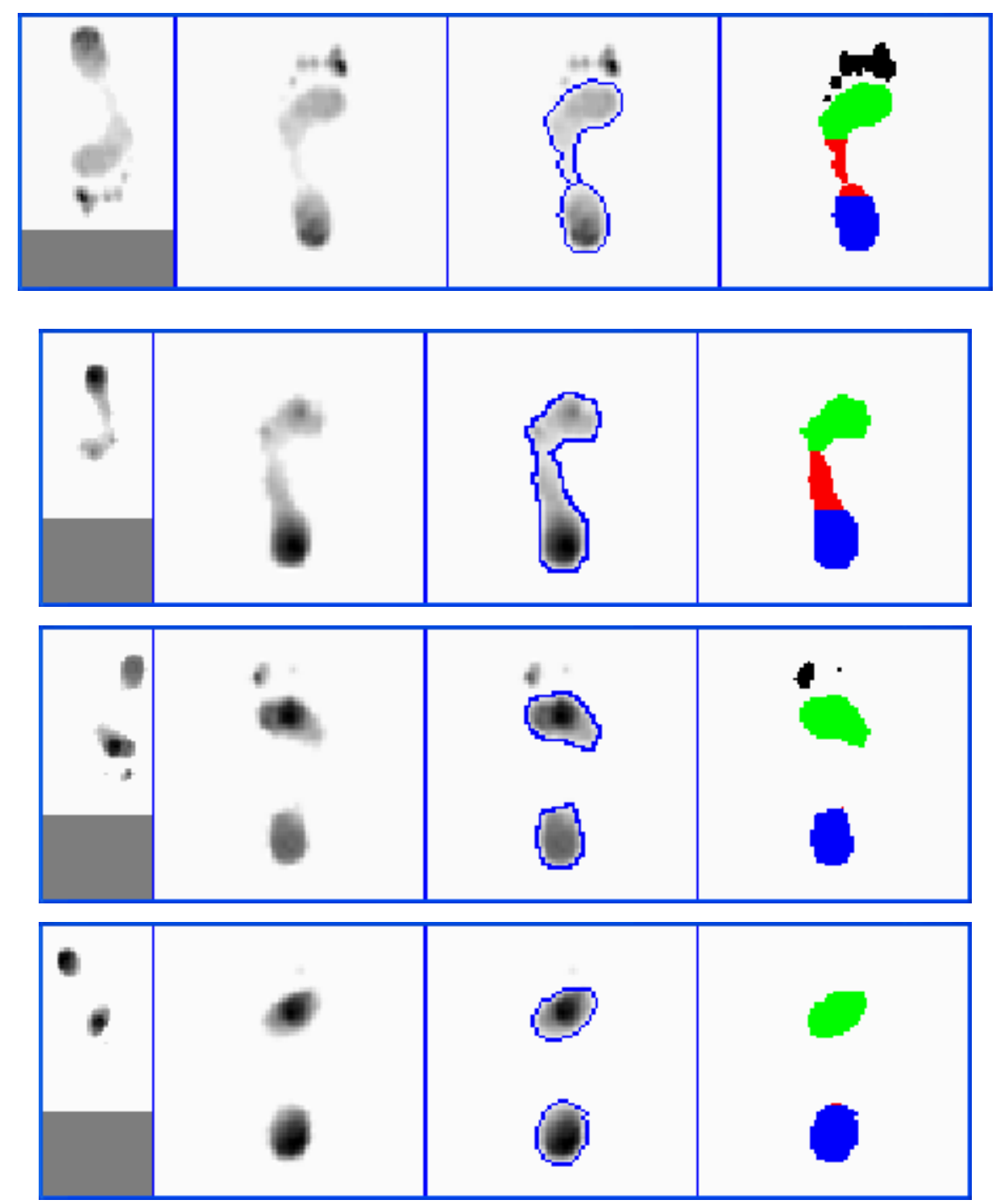

Figure 5 